\title{
SACARIFICACIÓN ENZIMÁTICA DE LA VAINA DE ARVEJA FRESCA (Pisum Sativum L.) VARIEDAD SABANERA PARA OBTENCIÓN DE AZÚCARES REDUCTORES
}

\author{
SACCHARIFICATION ENZYMATIC APPLIED TO \\ THE FRESH PEA POD VARIETY PISUM SATIVUM $L$. \\ FOR OBTAIN REDUCING SUGAR.
}

\author{
Simón Andrés González González ${ }^{1}$
}

\section{RESUMEN}

En este estudio se describe el proceso de sacarificación enzimática aplicada a la vaina de arveja fresca, variedad Santa Isabel, material vegetal rico en contenido lignocelulósico que puede ser aprovechado para la obtención de azúcares. Para efectos de la investigación se planteó aplicar nueve tratamientos, teniendo en cuenta: el tipo de solvente (agua desionizada y ácido sulfúrico), el tipo de enzima (Novozymes ${ }^{\circledR}$ y Alkozym $^{\circledR}$ ), la relación de dilución, tanto del solvente como de la enzima y el uso o no de autoclave como parte del tratamiento aplicado a las muestras. La determinación cualitativa y cuantitativa de los azúcares se realizó por cromatografía líquida de alta resolución (HPLC) utilizando un equipo LC-2010 AHT Shimadzu ${ }^{\circledR}$, con un detector de índice de refracción RID-10A Shimadzu ${ }^{\circledR}$ y una columna Shodex SC1011 ${ }^{\circledR}$, usando agua desionizada como fase móvil.

Se realizó una caracterización química de la vaina de arveja, determinando porcentajes de humedad, proteína, fibra, grasa y cenizas y análisis de lignina, holocelulosa, celulosa y hemicelulosa. Para el estudio estadístico del para el contenido de azúcares se llevó a cabo un análisis de varianza y una comparación de medias a través de la prueba de Tukey, con el fin de hallar el mejor tratamiento, encontrándose que el tratamiento 8 , es el de mejores resultados para la obtención de glucosa y fructosa; considerando el no uso de ácido sulfúrico, el mejor tratamiento sería el 4, el cual aplica para los tres azúcares identificados y cuantificados (sacarosa, glucosa y fructosa). A los datos obtenidos de los análisis de lignina, holocelulosa, celulosa y hemicelulosa se les aplicó un análisis de varianza con el fin de establecer si las muestras sometidas a los tratamientos de hidrólisis presentan una diferencia significativa en contenido de material lignocelulósico.

Palabras clave: enzima, hidrólisis, hidrolizado, material lignocelulósico, sacáridos.

\footnotetext{
1 Programa de Ingeniería de alimentos, Fundación Universitaria Agraria de Colombia. Colombia, andriu-115@hotmail.com.
} 


\begin{abstract}
This study describes the process of enzymatic saccharification applied to the fresh pea pod variety Santa Isabel, a content rich vegetable material lignocellulose, which can be exploited to obtain sugars. For effects of the research they thought about to apply nine treatments keeping in mind: the solvent type (deionized water and sulfuric acid), the enzyme type (Novozymes ${ }^{\circledR}$ and Alkozym ${ }^{\circledR}$ ), the so much dilution relationship of the solvent like of the enzyme, one also kept in mind the use or not of autoclave like part of the treatment applied to the samples. The qualitative and quantitative determination of the sugars was carried out for liquid chromatography of high resolution (HPLC) using a team LC-2010 AHT Shimadzu ${ }^{\circledR}$, with a detector of refraction index RID-10A Shimadzu ${ }^{\circledR}$ and a column Shodex SC1011, using deionized water as the phase mobile. Similarly it was a chemical characterization of the pea pods certain percentage of moisture, protein, fiber, fat and ash. It also performed analysis of Lignin, holocellulose, cellulose and hemicellulose. The Statistical analysis for the content of sugars was performed by analysis of variance and comparison of means by Tukey test, in order to find the best treatment; Finding that treatment 8, the works best for obtaining glucose and fructose, but considering not to use sulfuric acid, the best treatment would be 4; which applies to all three sugars identified and quantified (sucrose, glucose and fructose). For data obtained from the analysis of lignin, holocellulose, cellulose and hemicellulose was applied to analysis of variance to establish whether the samples subjected to hydrolysis treatments show a significant difference in content of lignocellulosic material.
\end{abstract}

Key words: enzyme, hydrolysis, hydrolyzed, lignocellulosic material, saccharides.

Aprobado: Junio 29 de 2010

\title{
INTRODUCCIÓN
}

La cáscara de arveja verde, materia orgánica proveniente de los desechos agrícolas de las plazas de mercado y de los centros de acopio de alta producción, se ha convertido en foco de contaminación por su rápida descomposición. Actualmente, este material es transportado con los demás residuos agrícolas hacia los rellenos sanitarios, donde genera lixiviados que contaminan los suelos y ríos a los que tenga acceso; de igual manera, la acumulación del material en los rellenos produce gases contaminantes por la descomposición de los desechos albergados en estos sitios, convirtiéndose no solo en un problema de contaminación ambiental, si no también, en problema de carácter social y de seguridad alimentaria, ya que muchas personas que viven en los alrededores subsisten y se alimentan de estos desechos.

Conocida esta problemática, muchas entidades se han dedicado a encontrar alternativas viables de solución, entre ellas, llevar los residuos vegetales a sitios apropiados donde se puedan descomponer de forma adecuada y utilizar este material para la elaboración de abonos orgánicos. Lamentablemente, la gran cantidad de dichos residuos que se obtiene en los centros de acopio, sobrepasa la capacidad instalada, razón por la cual, parte de estos, se siguen 
depositando en los rellenos sanitarios. Sin embargo, se han planteado algunas alternativas de uso para este tipo de material, resultante de los residuos de cosecha, al igual que para la parte no comestible de frutas y verduras que poseen un sin número de componentes, tales como, las cortezas de cítricos de donde se extrae pectina; colorantes y aceites esenciales que se obtienen de algunas frutas y hortalizas y, de cáscaras de maracuyá, fibra dietaría con múltiples aplicaciones Mejía, et al. (2007) "Aprovechamiento del residuo agroindustrial de mango común (Mangifera indica L.), para la obtención de azúcares fermentables”, y Lázaro, L. y Arauzo, P. (1994) "Aprovechamiento de residuos de la industria de conservas vegetales. Hidrólisis enzimática".

En la investigación se planteó aplicar tratamientos de hidrólisis enzimática combinados con otros tipos de hidrólisis, con el fin de obtener azúcares reductores, como una alternativa de uso de los residuos generados, para minimizar así el impacto que estos provocan al ambiente. Triana (2010) aplicó tratamientos de hidrólisis a residuos de cosecha del cultivo de café no solo para la obtención de azúcares, si no en la fermentación del hidrolizado para la obtención de etanol. En Centroamérica, autores como Carballo et al. (2006) proponen aplicar tratamientos de hidrólisis en maderas con el fin de obtener alcohol carburante y de encontrar las mejores maderas para este tipo de procesos.

Según la FAO, en el 2008, la producción mundial de arveja verde (incluidos los guisantes) fue de 8,4 millones de toneladas. En Colombia, de acuerdo con la Federación de Cultivadores de Cereales y Leguminosas Fenalce (2010), se obtuvo en el 2008 una producción estimada de arveja de 80 mil toneladas para consumo en fresco. Se estima que por cada tonelada de arveja producida, su vaina en peso fresco representa $450 \mathrm{~kg}$., lo que indica que aproximadamente el 45\% del cultivo de arveja es residuo agroindustrial en forma de vaina (Hurtado, 2005). En sitios como los centros de acopio, la vaina de arveja es uno de los residuos agrícolas más representativo después de la hoja de mazorca y los sobrantes de las hortalizas. Por esta razón, es conveniente buscar una opción de manejo de este tipo de material, en donde se plantee una alternativa viable de uso, mediante la que se logren minimizar los problemas que causa el mal manejo de los residuos sólidos. En este caso, aprovechando que la vaina de arveja fresca contiene abundante material lignocelulósico, se puede pensar en aplicar tratamientos de hidrólisis que sobrepasen la capa protectora de lignina y faciliten el acceso a las moléculas de celulosa, las degrade y se obtenga azúcares que se puedan identificar, caracterizar y cuantificar, que permita darle un uso y un valor agregado a este tipo de material.

\section{MÉTODOS}

El trabajo se realizó en dos fases: en la primera se efectúo la caracterización química de la vaina de arveja y en la segunda se realizaron los tratamientos de hidrólisis, análisis de los azúcares y composición lignocelulósica. 


\section{Fase 1: Caracterización química de vaina de arveja verde variedad Santa Isabel}

Para los siguientes análisis se trabajó con vaina de arveja verde, variedad Santa Isabel, comúnmente denominada Sabanera, proveniente de la Central de Abastos "Corabastos", Bogotá. Las vainas de arveja fueron picadas y colocadas en bandejas plásticas que se ubicaron en estufa de desecación a $50^{\circ} \mathrm{C}$ por 5 días; se maceró la muestra para disminuir el tamaño. En esta fase, todos los análisis se hicieron por triplicado y los resultados se reportan en base seca; se hizo análisis de humedad, grasa (extracto etéreo), fibra, cenizas, proteína, lignina, holocelulosa y celulosa (Ver Tabla1).

Tabla 1. Análisis realizados a la vaina de arveja fresca ${ }^{2}$

\begin{tabular}{|l|l|}
\hline \multicolumn{1}{|c|}{ Análisis } & \multicolumn{1}{c|}{ Método } \\
\hline Humedad & Gravimétrico, termobalanza \\
\hline Fibra & Fibra cruda (A.O.A.C 962.09/90)1 \\
\hline Proteína & Método micro-Kjeldahl2 \\
\hline Grasa (extracto etéreo) & Extracción Soxhlet 3 \\
\hline Cenizas & A.O.A.C. 942.054 \\
\hline Lignina & Lignina Klason5 \\
\hline Holocelulosa & Clorinación 6 \\
\hline Celulosa & Norma ASTM 1695-777 \\
\hline Hemicelulosa & Cálculo matemático, por diferencia. \\
\hline Azúcares & Cromatografía HPLC \\
\hline
\end{tabular}

Fuente: el autor.

\section{Fase 2: Tratamientos de hidrólisis, análisis de azúcares y composición ligninocelulósica}

Los tratamientos aplicados (ver tabla 2) se plantearon teniendo en cuenta la correlación de las variables de criterio y de control establecidas:

2 Norma descrita en Bernal de Ramírez, 1993

Descrito en Bernal de Ramírez, 1993 y Nielsen, 2007

Método descrito por Bernal de Ramírez, 1993 y por Min, 2009

Norma descrita en Bernal de Ramírez, 1993

Descrito en Gumeta, 2009. y Sánchez, 2010

Descrito por Wise 1946, Browing 1967 y González, 2010

Mencionada por Triana, 2010 y Tortosa, 2007. 
Tabla 2. Tratamientos de hidrolisis aplicados

\begin{tabular}{|c|l|c|c|}
\hline Tratamiento & \multicolumn{1}{|c|}{ Solvente } & Autoclave & Enzima \\
\hline 1 & Agua Desionizada & SI & ALKOZYM \\
\hline 2 & Ácido sulfúrico-Agua Desionizada & SI & ALKOZYM \\
\hline 3 & Agua Desionizada & NO & ALKOZYM \\
\hline 4 & Agua Desionizada & SI & NOVOZYMES \\
\hline 5 & Ácido sulfúrico-Agua Desionizada & SI & NOVOZYMES \\
\hline 6 & Agua Desionizada & NO & NOVOZYMES \\
\hline 7 & Agua Desionizada & SI & SIN ENZIMA \\
\hline 8 & Ácido sulfúrico-Agua Desionizada & SI & SIN ENZIMA \\
\hline 9 & Agua Desionizada & NO & SIN ENZIMA \\
\hline
\end{tabular}

Fuente: el autor.

Variables de criterio: tipo de solvente para realizar la hidrólisis y tipo de enzima para usar en cada tratamiento

Variables de control: relación solución- muestra (10/1), presión 15 psi, tiempo en autoclave 1 hora, relación enzima- muestra (1/10).

Variables de respuesta: contenido de azúcares.

\section{Aplicación de tratamientos de hidrólisis}

Se tomaron frascos para autoclave de $250 \mathrm{~mL}$, numerados de 1 a 9; en cada uno se colocaron $10 \mathrm{~g}$ de vaina de arveja seca y macerada; luego, se añadió agua y ácido sulfúrico, según el tratamiento, como se indica en la Tabla 2. Cada muestra se diluyó en $100 \mathrm{~mL}$ de agua desionizada (tratamientos solo agua) y para los tratamientos ácido sulfúrico-agua desionizada. Se diluyó cada muestra en $100 \mathrm{~mL}$ de agua desionizada y 0,5 $\mathrm{mL}$ de ácido sulfúrico [97\%]. Cada tratamiento se realizó por triplicado.

Las muestras pertenecientes a los tratamientos $1,2,4,5,7$ y 8 se colocaron en el autoclave a una presión 15 psi durante una hora; transcurrido este tiempo se sacaron del autoclave, dejándolas enfriar para medir su pH; con la adición de hidróxido de sodio, se neutralizó y se enrazó hasta un volumen de $250 \mathrm{~mL}$. A todas las muestras se les adicionó $1 \mathrm{ml}$ de enzima para luego colocarlas en incubación a $45^{\circ} \mathrm{C}$, durante una hora. Para los tratamientos 3, 6 y 9 se procedió de la misma manera, omitiendo el proceso de esterilización en autoclave. Realizada la incubación, se filtraron las muestras en embudos de decantación y papel filtro de $110 \mathrm{~mm}$; el sólido se secó y se reservó para los análisis de lignina, holocelulosa y celulosa. De la parte líquida, se tomaron $36 \mathrm{~mL}$ de muestra por cada frasco repartiéndolos en tres tubos Falcon; se centrifugaron por 20 minutos y luego se transfirió el líquido a otro tubo con el fin de retirar el sólido precipitado. Inmediatamente, se realizó el acondicionamiento de la muestra 
para el análisis en el equipo de cromatografía líquida de alta presión (HPLC). Se identificaron estrictamente los viales (limpios y secos); para colocar la muestra dentro del vial se usó una jeringa de $5 \mathrm{ml}$ acondicionada con un porta membranas que contiene una membrana de filtración analítica en Fluoruro de polivinilideno (PVDF) de $0.45 \mu \mathrm{m}$. Las muestras se llevaron a refrigeración hasta el momento de ser ubicadas en la bandeja del cromatógrafo.

En esta investigación se eligieron dos tipos de enzimas: la Alkozym CPX A ${ }^{\circledR}$, que se seleccionó por recomendación del fabricante, y la Novozymes ${ }^{\circledR}$ (NS50013 y NS50010) que se eligió por ser una enzima trabajada, con buenos resultados en (Ying, 2008) y (Bettín \& Quintero, 2010).

\section{Análisis de lignina, holocelulosa y celulosa (después del tratamiento de hidrólisis)}

Se llevó a cabo el mismo procedimiento que se utilizó para las muestras analizadas, antes de aplicar el tratamiento de hidrólisis (ver tabla 1). Para el análisis de los datos se realizó una comparación entre varianzas con el fin de establecer si hay o no diferencia significativa en el contenido del material lignocelulósico, después de haber sometido las muestras a los diferentes tratamientos.

\section{Análisis de azúcares}

Para este análisis se usó un equipo de cromatografía líquida de alta presión (HPLC) LC2010AHT Shimadzu ${ }^{\circledR}$, con un detector de índice de refracción RID-10A Shimadzu ${ }^{\circledR}$ y una columna Shodex SC1011; las características de esta columna se encuentran en Shodex 1, (2011). Las condiciones con las cuales se elaboró el método de análisis fueron las siguientes ( ver tabla 3).

Tabla 3. Método usado para análisis de las muestras en el HPLC

\begin{tabular}{|l|l|}
\hline Tiempo de corrida por muestra & $30 \mathrm{~min}$ \\
\hline Fase móvil & Agua Desionizada $100 \%$ \\
\hline Flujo & $0.75 \mathrm{~mL} /$ min \\
\hline Temperatura del horno & $59^{\circ} \mathrm{C}$ \\
\hline Volumen de la muestra & $20 \mu \mathrm{L}$ \\
\hline
\end{tabular}

Fuente: Autor, modificado (Shodex 1, 2010).

\section{Identificación, interpretación y análisis de los gráficos obtenidos en el HPLC}

Según la información encontrada en Urbaneja (1997), Ying (2008) y Cuervo (2009) sobre el contenido lignocelulósico en material vegetal y los tratamientos de hidrólisis para obtención de azúcares, se presume que el hidrolizado de vaina de arveja puede estar compuesto de una cantidad significativa de sacarosa, glucosa y fructosa, razón por la cual se procedió a elaborar curvas de calibración con estándares para estos tres azúcares, aplicando el mismo método de 
análisis que se utilizó para analizar las muestras de interés, logrando su tiempo de retención y el orden en que aparecen los picos correspondientes a cada azúcar y calcular la concentración de estos azúcares en las muestras, mediante las siguientes expresiones:

$$
\begin{aligned}
& \text { [] Sacarosa } y=216,79 x(1) \\
& \text { [] Glucosa } y=187,73 x(2) \\
& \text { [] Fructosa } y=176,15 x(3)
\end{aligned}
$$

Donde y corresponde al área bajo la curva de pico correspondiente para cada azúcar y $x$ la concentración en parte por millón (ppm) de cada azúcar. Con esta información y la compilación de datos (áreas bajo la curva de cada pico) se calculó la concentración expresada en miligramo de azúcar (sacarosa, glucosa o fructosa) por gramo de muestra usando las expresiones:

$$
\begin{aligned}
& \frac{m g \text { de sacarosa }}{g \text { de muestra }}=\frac{y * 0,00461276 * 0,25}{m a} \\
& \frac{m g \text { de giucosa }}{g \text { de muestra }}=\frac{y * 0,0053268 * 0,25}{m \cdot a} \\
& \frac{m g \text { de fructosa }}{g \text { de muestra }}=\frac{y * 0,00566524 * 0,25}{m a}
\end{aligned}
$$

En este caso, y corresponde al área bajo la curva de pico correspondiente para cada azúcar; 0,25 es el volumen de dilución de la muestra, expresado en litros; m.a es el peso en gramos de la muestra inicial. En seguida, se tabularon los datos para proceder a aplicar el análisis de varianza y la comparación de medias a través de la prueba de Tukey $(\alpha 0.05)$ entre los diferentes tratamientos y sus variables con el fin de encontrar los mejores tratamientos.

\section{RESULTADOS Y ANÁLISIS}

\section{Caracterización química}

La tabla 4 muestra la composición de la vaina de arveja fresca, determinada en el laboratorio; los valores de cenizas, fibra, grasa y proteína están reportados con base en el peso de la muestra seca. Como se puede apreciar, el contenido de fibra es alto, lo que demuestra que este material presenta buenas condiciones para ser utilizado en la obtención de azúcares fermentables; de igual manera, al analizar el contenido proteína, el 13,34\% de proteína en base seca, representa el 1,84\% de proteína en base húmeda; es decir, por cada 100 $\mathrm{g}$ de vaina de arveja fresca, 1,84 g son proteína, siendo este valor bastante bajo, comparado con el 5\% de proteína Kehr y Mera (2007) que contiene la variedad comestible sugar snap. 
Tabla 4. Caracterización química vaina de arveja fresca variedad Santa Isabel

\begin{tabular}{|l|c|c|}
\hline \multicolumn{1}{|c|}{ Composición } & Valor promedio hallado (\%) & Desviación estándar \\
\hline Humedad inicial ( vaina fresca) & 86,22 & 1,1890 \\
\hline Humedad después de secado & 2,53 & 0,2312 \\
\hline Cenizas & 4,65 & 0,0833 \\
\hline Fibra & 55,07 & 1,4317 \\
\hline Grasa & 1,64 & 0,5314 \\
\hline Proteína & 13,34 & 1,9044 \\
\hline
\end{tabular}

Fuente: el autor.

En la figura 1 se muestran los porcentajes promedio de lignina, holocelulosa, celulosa y hemicelulosa hallados en la vaina de arveja después de haber aplicado los diferentes tratamientos de hidrólisis. Los porcentajes están calculados sobre la cantidad de muestra seca usada en cada tratamiento. Igualmente en la tabla 5, se muestra la interpretación del análisis de varianza aplicado con la intención de comparar y encontrar una diferencia significativa entre los diferentes tratamientos.

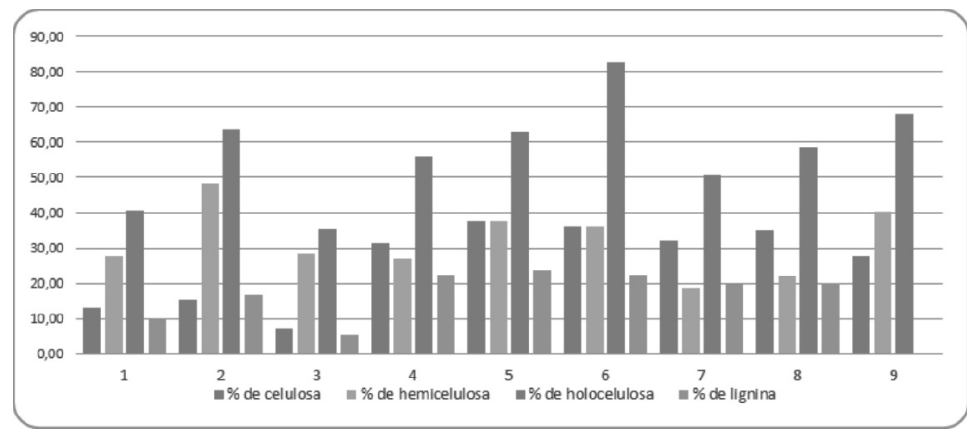

Figura 1. Contenido de material lignocelulósicos para los nueve tratamientos manejados

\section{Análisis de varianza}

En la tabla 5 se presentan los diferentes análisis de varianza realizados para la lignina, holocelulosa, celulosa y hemicelulosa para cada uno de los nueve tratamientos. 
Tabla 5. Resumen análisis de varianza

\begin{tabular}{|c|c|c|c|}
\hline prueba & Tratamientos a comparar & $\begin{array}{c}\text { ¿Hay diferencia } \\
\text { significativa? }\end{array}$ & $\mathbf{p}$ \\
\hline \multirow{7}{*}{ Lignina } & con enzíma Alkozym & si & 0,00109111 \\
\hline & con enzíma novozymes & no & 0,91468248 \\
\hline & sin enzíma & no & 0,86204355 \\
\hline & con agua HPLC en autoclave & si & 0,0006286 \\
\hline & con agua-acido en autoclave & si & 0,00410031 \\
\hline & con agua HPLC sin autoclave & no & 0,06399717 \\
\hline & todos & si & 0,00178723 \\
\hline \multirow{7}{*}{ Holocelulosa } & con enzíma Alkozym & si & 0,0116672 \\
\hline & con enzíma novozymes & si & 0,00025861 \\
\hline & sin enzíma & no & 0,11382739 \\
\hline & con agua HPLC en autoclave & no & 0,13789925 \\
\hline & con agua-acido en autoclave & no & 0,0747503 \\
\hline & con agua HPLC sin autoclave & si & 0,00311167 \\
\hline & todos & si & 2,7259E-05 \\
\hline \multirow{7}{*}{ Celulosa } & con enzíma Alkozym & si & 0,03094564 \\
\hline & con enzíma novozymes & si & 0,0006362 \\
\hline & sin enzíma & no & 0,0953523 \\
\hline & con agua HPLC en autoclave & si & 0,00889057 \\
\hline & con agua-acido en autoclave & si & 0,0002103 \\
\hline & con agua HPLC sin autoclave & si & $6,0622 \mathrm{E}-05$ \\
\hline & todos & si & $8,5731 \mathrm{E}-08$ \\
\hline \multirow{7}{*}{ Hemicelulosa } & con enzíma Alkozym & si & 0,04569827 \\
\hline & con enzíma novozymes & si & 0,00957996 \\
\hline & sin enzíma & si & 0,03514097 \\
\hline & con agua HPLC en autoclave & no & 0,38057655 \\
\hline & con agua-acido en autoclave & si & 0,00279746 \\
\hline & con agua HPLC sin autoclave & si & 0,0443841 \\
\hline & todos & si & 0,00056475 \\
\hline
\end{tabular}

Fuente: el autor. 
Inicialmente, se compararon los nueve tratamientos, y se encontró una diferencia significativa en el contenido de lignina, holocelulosa, celulosa y hemicelulosa, lo que indica que el nivel de degradación fue distinto en cada uno de ellos.

Según el análisis de varianza realizado para los datos obtenidos en los tratamientos con enzima Alkozym, se encontró una diferencia significativa en el contenido de lignina, holocelulosa, celulosa y hemicelulosa entre los tres tratamientos a los cuales se les aplicó esta enzima, indicando que el nivel de degradación provocado por la hidrolisis no es el mismo en los tres tratamientos analizados; de igual manera, se puede evidenciar que el contendido de lignina es menor en estos tres tratamientos, si se compara con los 6 restantes, revelando una probable mayor influencia de estos en la degradación de la lignina. Igualmente, ocurre con el contenido de celulosa en donde se aprecia aproximadamente un 15\% menos en contenido, comparado con los demás tratamientos, lo que permite creer que hubo mayor obtención de azúcares. Esta afirmación entra a discusión más adelante en el análisis de contenido de azúcares.

No se encontró una diferencia significativa en el contenido de lignina en los tratamientos a los que se les aplicó la enzima Novozymes, mientras que sí hay una diferencia significativa en el contenido de holocelulosa, celulosa y hemicelulosa, lo que permite pensar, que para estos tres tratamientos, el nivel de degradación generado por la hidrólisis, afectó en menor proporción la lignina presente en las muestras y, a su vez, el nivel de degradación de la holocelulosa, celulosa y hemicelulosa se presentó en mayor proporción, pero no se obtuvo el mismo resultado en cada uno de estos tratamientos.

De otra parte, no se encontró en los tratamientos a los que no se les aplicó enzima, una diferencia significativa en el contenido de lignina, holocelulosa y celulosa, mientras que en el contenido de hemicelulosa, sí hay diferencia significativa. De igual manera, se compararon los tratamientos que se trabajaron con agua desionizada en autoclave, encontrando que hay una diferencia significativa en el contenido de lignina y celulosa, mientras que no se identificó una diferencia significativa en el contenido de holocelulosa y hemicelulosa. Una segunda comparación entre los tratamientos con agua desionizada-acido sulfúrico en autoclave, arrojó que estos tratamientos presentan una diferencia significativa en el contenido de lignina, celulosa y hemicelulosa y una diferencia no significativa en el contenido de holocelulosa. También, se compararon los tratamientos con agua desionizada sin autoclave, arrojando como resultado una diferencia significativa en el contenido de holocelulosa, celulosa, hemicelulosa, mientras que en el contenido de lignina, una diferencia no significativa.

Mejía (2007) en su trabajo sobre hidrólisis en cáscara de mango, reporta datos sobre el contenido de celulosa y hemicelulosa de las muestras tratadas con cada uno de los tratamientos aplicados y en las muestras sin tratamientos, encontrando que estas últimas fueron las de mayor contenido, resultado lógico, puesto que si se somete el material vegetal a un proceso de hidrólisis, este sufre una degradación que altera su composición inicial. 
En la figura 1 se puede apreciar el comportamiento del contenido de material lignocelulósico en las muestras de arveja, notándose que no se presenta un comportamiento como el mencionado anteriormente, a pesar de observar que las muestras sufrieron degradación. Esta apreciación no es generalizada, mientras que en los tratamientos 1, 2 y 3 se nota una disminución en el contenido de material lignocelulósico; en los demás tratamientos no se observa una diferencia muy marcada.

\section{Análisis de los hidrolizados}

La observación de los hidrolizados permitió identificar ciertas características en cuanto al color del líquido, aroma, turbidez y color de residuo. Las muestras pertenecientes a los tratamientos con agua desionizada, con enzima y en autoclave presentaron un aroma similar al del jugo de caña, mientras que las muestras de los tratamientos con agua desionizada - ácido sulfúrico, enzima y autoclave presentaron un olor a hierbas aromáticas; de igual manera, las muestras pertenecientes a los tratamientos con agua desionizada, con enzima y sin autoclave presentaban un aroma a miel de caña. A las muestras que simplemente se diluyeron en agua desionizada, no se les aplicó enzima y no se llevaron a autoclave y presentaron un aroma a vegetales frescos. Los hidrolizados también presentaron diferencias en cuanto al color, resaltando los hidrolizados que se trabajaron con ácido sulfúrico, los cuales presentaban un color más oscuro mientras que los de color más claro fueron los que no se llevaron a la autoclave.

Al igual que el hidrolizado, el residuo sobrante de la hidrólisis también presenta unas características en cuanto a apariencia y color. En la figura 2 se puede observar que la imagen b la cual pertenece a un tratamiento de agua desionizada - ácido sulfúrico, presenta una tonalidad más oscura que las otras dos muestras, donde se nota una mayor degradación, debido a la utilización de ácido.

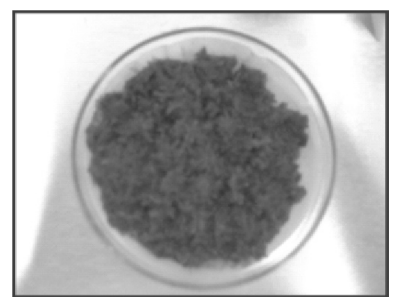

(a)

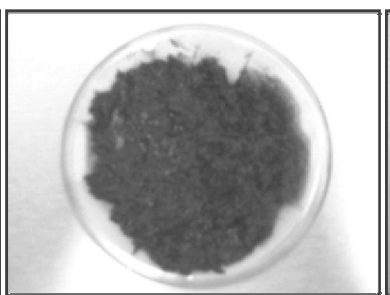

(b)

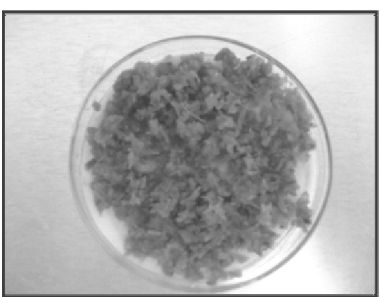

(c)

Figura 2. Diferencia en apariencia del residuo sólido después de hidrólisis. (a) Tratamiento agua desionizada en autoclave, (b) Tratamiento con ácido, (c) Tratamiento agua desionizada sin autoclave. 
El análisis de los hidrolizados permitió obtener un cromatograma por cada muestra analizada, reportando un tiempo de retención y un área bajo la curva para el pico correspondiente a cada uno de los azúcares identificados. En la tabla 6 se muestra el contenido promedio en miligramos de sacarosa, glucosa y fructosa por gramo de muestra analizada, reportado para los nueve tratamientos manejados.

Tabla 6. Miligramos de sacarosa, glucosa y fructosa promedio por muestra cuantificados para cada tratamiento

\begin{tabular}{|c|c|c|c|}
\hline Tratamiento & $\begin{array}{c}\text { mg de sacarosa/g } \\
\text { de muestra }\end{array}$ & $\begin{array}{c}\text { mg de glucosa/g } \\
\text { de muestra }\end{array}$ & $\begin{array}{c}\text { mg de fructosa/ } \\
\text { de muestra }\end{array}$ \\
\hline 1 & 3,88120718 & 49,0930304 & 26,4353438 \\
\hline 2 & 17,7087815 & 73,1960284 & 27,8748692 \\
\hline 3 & 4,78804244 & 51,3096767 & 47,1539595 \\
\hline 4 & 29,3497755 & 188,686297 & 76,6282377 \\
\hline 5 & 272,749645 & 220,016877 & 70,8900065 \\
\hline 6 & 24,7289963 & 144,491556 & 67,3271941 \\
\hline 7 & 3,04970118 & 25,1648998 & 9,77726344 \\
\hline 8 & 268,971596 & 223,89238 & 80,8818984 \\
\hline 9 & 33,2725366 & 147,641103 & 43,7628072 \\
\hline
\end{tabular}

Fuente: el autor.

En la tabla anterior se puede observar que los tratamientos que presentan mayor contenido de sacarosa son el 4, 8 y 5 en su orden, para los cuales hay una serie de observaciones que se mencionan en el análisis de datos de la prueba de Tukey. En cuanto al contenido de glucosa, los tratamientos con mayor concentración son el 4, 5 y 8; así mismo, para el contenido de fructosa, sobresalen los tratamientos 4, 5, 6 y 8. Los picos correspondientes a sacarosa, glucosa y fructosa se identificaron por sus tiempos de retención y el volumen de elución, usando como referencia los cromatogramas de los estándares y los volúmenes de elución reportados por Shodex 2 (2011), en un análisis de sacáridos a condiciones óptimas de trabajo para la columna utilizada $\left(80^{\circ} \mathrm{C}\right.$ y $\left.1 \mathrm{ml} / \mathrm{min}\right)$, (ver Tabla 7$)$.

Tabla 7. Volumen de elución y tiempo de retención para sacarosa, glucosa, fructosa

\begin{tabular}{|l|c|c|}
\hline \multicolumn{1}{|c|}{ Sacárido } & Volumen de elución $(\mathbf{m L})$ & Tiempo de retención $(\mathbf{m i n})$ \\
\hline Sacarosa & 6.29 & 6.29 \\
\hline glucosa & 7.30 & 7.30 \\
\hline Fructosa & 8.85 & 8.85 \\
\hline
\end{tabular}

Fuente: Shodex. 
Una característica particular de todos los cromatogramas obtenidos es que aparece una serie de picos sin identificar entre los 5 y 7 minutos de corrida antes del pico de sacarosa, que según Takashi Kotsuka General Manager de Shodex ${ }^{\circledR}$, probablemente son oligosacáridos, estructuras de tres a diez azúcares; para su análisis, se recomienda utilizar una columna que permita una mejor separación e identificación de estos oligosacáridos o se lleven a cabo ensayos con mayor tiempo de sacarificación, modificando el tiempo de exposición con la enzima, con el fin de llegar a azúcares menos complejos. Dentro de la lista de posibles compuestos se encuentran los sacáridos de la tabla 8, que por su volumen de elución y naturaleza son los sacáridos que posiblemente están presentes en los hidrolizados. De este listado, se estima que los sacáridos con mayor probabilidad son la rafinosa, un trisacárido compuestos por galactosa y sacarosa presente en algunas leguminosas Badui (2006) y la estaquiosa compuesta por rafinosa y galactosa que también está presente en muchos vegetales (Nakakuki, T. 2002).

Según Mejía (2007), puede existir la posibilidad que parte del material hidrolizado se convierta en compuestos diferentes a sacáridos tales como ácidos orgánicos, compuestos aromáticos, furfural e hidroximetilfurfural, los cuales podrían ser causantes de inconvenientes en caso de usar los hidrolizados para producción de etanol; es decir, que la hidrólisis del material lignocelulósico no necesariamente conduce a obtención de azúcares.

Tabla 8. Lista de posibles oligosacáridos presentes en el hidrolizado de arveja

\begin{tabular}{|l|c|}
\hline \multicolumn{1}{|c|}{ Sacáridos } & Volumen de elución $\mathbf{( m L )}$ \\
\hline Gentiobiosa & 6.08 \\
\hline Isomaltosa & 6.26 \\
\hline Isomaltotriosa & 5.75 \\
\hline 1- kestosa & 5.75 \\
\hline Kojibiosa & 6.21 \\
\hline Maltotriosa & 5.89 \\
\hline Nystose & 5.45 \\
\hline Panosa & 5.78 \\
\hline D (+)- Raffinosa & 5.78 \\
\hline Stachyose & 5.57 \\
\hline
\end{tabular}

Fuente: Shodex 2.

\section{Influencia de la combinación de tratamientos de hidrólisis en la producción de azúcares}

De acuerdo con Lázaro y Arauzo (1994) el uso de un tratamiento con ácido puede disminuir el efecto protector de la lignina y favorecer el acceso a la celulosa y la degradación enzimática; de igual manera, explica en un esquema de producción de glucosa a partir de maíz, que la aplicación de hidrólisis ácida se utiliza para obtención de glucosa y la hidrolisis acido- 
enzimática, para la de fructosa. A si mismo Mejía (2007) manifiesta que la combinación de tratamientos (hidrólisis ácida, térmica y enzimática) en la producción de azúcares reductores, se puede ver afectada por la alta concentración del ácido usado en la hidrolisis y alcanza a degradar los azúcares que potencialmente pueda producir la hidrólisis enzimática, pero que al igual una adecuada combinación de los tratamientos, manejando un orden, un tiempo de exposición y una temperatura adecuada favorece a la producción de metabolitos fermentables. Lo mencionando anteriormente, explica por qué cada paquete de gráficos correspondiente a un mismo tratamiento muestra un esquema similar. En los tratamientos con Alkozym se observan, además de los picos de sacarosa, glucosa y fructosa, tres picos importantes antes de la sacarosa: uno de ellos de una altura considerable que puede indicar la presencia de un sacárido en una concentración importante. Picos, que según el tipo de solvente usado, presentan una altura diferente, lo que hace inferir que la forma de acción enzima varía según el tipo de solvente con el cual se trabaja la hidrólisis. Esta variación se puede corroborar con el análisis de media practicado al grupo de datos de los cuales se hará mención más adelante.

Algunos cromatogramas pertenecientes a los tratamientos con Novozymes ${ }^{\circledR}$ presentan un pico intermedio entre los picos de sacarosa y glucosa que probablemente es un anómero de glucosa, que por efecto de la temperatura, logró separarse. De igual manera, los cromatogramas, correspondientes al tratamiento 8, presentan un pico en el tiempo de retención correspondiente a sacarosa con un área considerable, que hace pensar que el pico de sacarosa no se encuentra o está sobrepuesto con picos adyacentes (ver figura 4), razón por la cual, el valor de área correspondiente a este pico no se tuvo en cuenta; caso similar sucedió en el tratamiento 5. Los tratamientos 7 y 8 presentan la particularidad de presentar un pico antes de la sacarosa con una altura considerable, que sobresale de los demás, indicando la presencia (con una concentración significativa) de un sacárido diferente a los identificados.

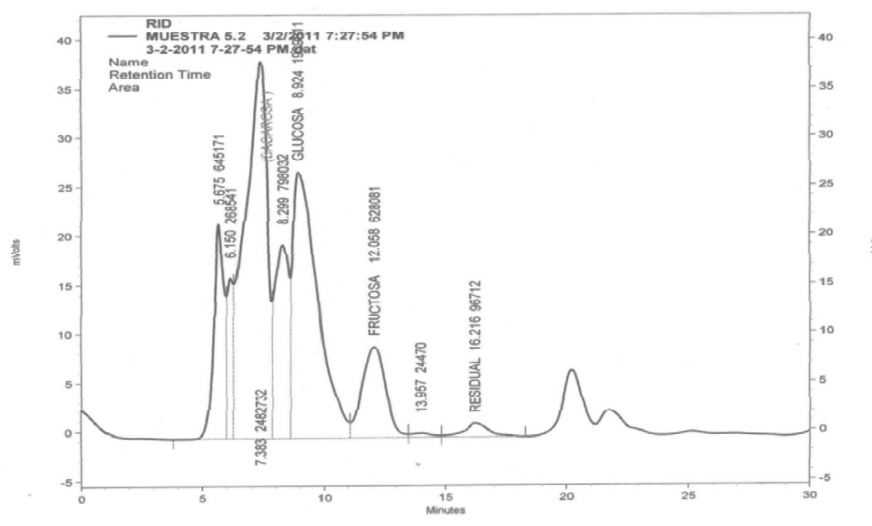

Figura 4. Cromatograma con pico de sacarosa enmascarado Fuente: el autor. 


\section{Análisis de tratamientos de hidrólisis según la comparación con la prueba de Tukey}

En las tablas 9, 10, 11,12 se muestran los resultados arrojados por la prueba de Tukey para las diferentes comparaciones realizadas, el agrupamiento señalado en las tablas hace referencia a que las medias con la misma letra no son significativamente diferentes.

Tabla 9. Comparación prueba de Tukey entre tratamientos

\begin{tabular}{|c|c|c|c|c|c|}
\hline Sacárido & Agr & ento & Media & $N$ & Tratamiento \\
\hline \multirow{9}{*}{ Sacarosa } & & A & 0,26897 & 1 & 8 \\
\hline & & B & 0,18062 & 5 & 5 \\
\hline & & $\mathrm{C}$ & 0,03327 & 8 & 9 \\
\hline & & $\mathrm{C}$ & 0,03179 & 7 & 6 \\
\hline & & $\mathrm{C}$ & 0,02935 & 9 & 4 \\
\hline & & $\mathrm{C}$ & 0,01992 & 8 & 2 \\
\hline & & $\mathrm{C}$ & 0,01089 & 8 & 1 \\
\hline & & $\mathrm{C}$ & 0,00539 & 8 & 3 \\
\hline & & $\mathrm{C}$ & 0,00305 & 9 & 7 \\
\hline \multirow{7}{*}{ Glucosa } & & A & 0,22389 & 9 & 8 \\
\hline & & A & 0,22002 & 9 & 5 \\
\hline & B & A & 0,18869 & 9 & 4 \\
\hline & B & & 0,14764 & 8 & 9 \\
\hline & B & & 0,14449 & 9 & 6 \\
\hline & & $\mathrm{C}$ & 0,08235 & 8 & 2 \\
\hline & & $\mathrm{C}$ & 0,07733 & 8 & 1 \\
\hline \multirow{2}{*}{ Glucosa } & & $\mathrm{C}$ & 0,05772 & 8 & 3 \\
\hline & & $\mathrm{C}$ & 0,02516 & 9 & 7 \\
\hline \multirow{9}{*}{ Fructosa } & & A & 0,080882 & 9 & 8 \\
\hline & & $\mathrm{A}$ & 0,080328 & 9 & 4 \\
\hline & & A & 0,07089 & 9 & 5 \\
\hline & B & A & 0,067327 & 9 & 6 \\
\hline & B & $\mathrm{C}$ & 0,053048 & 8 & 3 \\
\hline & & $\mathrm{C}$ & 0,05023 & 8 & 9 \\
\hline & & $\mathrm{D}$ & 0,031359 & 8 & 2 \\
\hline & & $\mathrm{D}$ & 0,027947 & 8 & 1 \\
\hline & & $\mathrm{E}$ & 0,009777 & 9 & 7 \\
\hline
\end{tabular}

Fuente: el autor. 
Tabla 10. Comparación prueba de Tukey para solventes usados

\begin{tabular}{|c|c|c|c|c|}
\hline Sacárido & Agrupamiento & Media & $\boldsymbol{N}$ & Solvente \\
\hline \multirow{3}{*}{ Sacarosa } & $\mathrm{A}$ & 0,0951 & 14 & Agua-ácido \\
\cline { 2 - 5 } & $\mathrm{B}$ & 0,01858 & 49 & Agua \\
\hline \multicolumn{5}{|c|}{} \\
\hline \multirow{2}{*}{ Glucosa } & $\mathrm{A}$ & 0,178998 & 26 & Agua-ácido \\
\cline { 2 - 5 } & $\mathrm{B}$ & 0,107581 & 51 & Agua \\
\hline \multicolumn{5}{|c}{} \\
\hline \multirow{2}{*}{ Fructosa } & $\mathrm{A}$ & 0,06285 & 26 & Agua-ácido \\
\cline { 2 - 5 } & $\mathrm{B}$ & 0,048367 & 51 & Agua \\
\hline
\end{tabular}

Fuente: el autor.

Tabla 11. Comparación prueba de Tukey según el uso de autoclave

\begin{tabular}{|c|c|c|c|c|}
\hline Sacárido & Agrupamiento & Media & $N$ & Autoclave \\
\hline \multirow{2}{*}{ sacarosa } & A & 0,042754 & 40 & SI \\
\hline & B & 0,023123 & 23 & NO \\
\hline \multirow{2}{*}{ Glucosa } & A & 0,138408 & 52 & SI \\
\hline & B & 0,117734 & 25 & $\mathrm{NO}$ \\
\hline \multirow[t]{2}{*}{ Fructosa } & A & 0,057287 & 25 & NO \\
\hline & B & 0,050987 & 52 & SI \\
\hline
\end{tabular}

Fuente: el autor.

Tabla 12. Comparación prueba de Tukey entre tipos de enzima

\begin{tabular}{|c|c|c|c|c|}
\hline Sacárido & Agrupamiento & media & $\boldsymbol{N}$ & Enzima \\
\hline \multirow{3}{*}{ Sacarosa } & $\mathrm{A}$ & 0,06618 & 21 & Novozymes \\
\cline { 2 - 5 } & $\mathrm{B}$ & 0,03126 & 18 & Sin enzima \\
\cline { 2 - 5 } & $\mathrm{B}$ & 0,01207 & 24 & Alkozym \\
\hline \multirow{4}{*}{ Glucosa } & $\mathrm{A}$ & 0,1844 & 27 & Novozymes \\
\cline { 2 - 5 } & $\mathrm{B}$ & 0,13164 & 26 & Sin enzima \\
\cline { 2 - 5 } & $\mathrm{C}$ & 0,07247 & 24 & Alkozym \\
\hline \multirow{3}{*}{ Fructosa } & $\mathrm{A}$ & 0,072848 & 27 & Novozymes \\
\cline { 2 - 5 } & $\mathrm{B}$ & 0,046838 & 26 & Sin enzima \\
\cline { 2 - 6 } & $\mathrm{C}$ & 0,037451 & 24 & Alkozym \\
\hline
\end{tabular}

Fuente: el autor. 
El análisis de varianza aplicado reveló que hay diferencia significativa entre los diferentes tratamientos aplicados en al contenido de los tres azúcares identificados. La prueba de Tukey permitió establecer que el tratamiento 7 es el menos apropiado ya que en los tres tipos de azúcares analizados presenta los menores valores de media.

Así mismo, se pudieron establecer los mejores tratamientos para la obtención de azúcares. En el caso de la sacarosa, se halló que el tratamiento 8 es significativamente diferente a los demás; pero como ya se había mencionado este tratamiento presenta inconvenientes en la resolución de los cromatogramas (se enmascaran o agrupan varios picos cercanos a la sacarosa), por lo que sería erróneo reportar tales picos como presencia de sacarosa. El tratamiento 5 también es significativamente diferente, pero al igual que el tratamiento 8 presenta inconvenientes con la resolución. Se determinó que el mejor tratamiento para la obtención de sacarosa es el 4.

En el caso de la glucosa, los resultados muestran que los mejores tratamientos fueron el 8 y 5, tratamientos en los que se usó ácido sulfúrico, y donde la purificación de estos hidrolizados se hizo más dispendiosa y costosa. El tratamiento 4, después de los tratamientos 8 y 5, presenta el mayor contenido de glucosa, siendo este el más conveniente. Para la fructosa se puede plantear la misma opción de la glucosa, donde los resultados presentan un comportamiento similar.

El análisis estadístico reporta una similitud entre los tratamientos en los que se usó ácido sulfúrico y agua; para los tres azúcares el valor de la media es mayor para los tratamientos de ácido sulfúrico siendo este valor significativamente diferente con respecto al valor de media de los tratamientos con solo agua. Caso similar ocurre con la comparación entre los tratamiento de las muestras con y sin autoclave.

Para el caso de la comparación entre tratamientos con distintos tipos de enzima, se encontró que el contenido medio de sacarosa en las muestras tratadas con Alkozym ${ }^{\circledR}$ y sin la enzima, no hay una diferencia significativa, mientras que los tratamientos en los que se usó Novozymes ${ }^{\circledR}$ presentan una diferencia significativa con respecto a los otros dos. En cuanto al contenido de glucosa y fructosa se observa una diferencia significativa entre los tipos de tratamientos; de igual manera, se puede establecer que la enzima Novozymes ${ }^{\circledR}$ es la que mejor actuó y la que presenta mayores valores de media en contenido de azúcares.

\section{CONCLUSIONES}

Según las consideraciones señaladas en el análisis de datos, el mejor tratamiento para la obtención de sacarosa, glucosa y fructosa sería el tratamiento 4 que trabaja con agua desionizada y con enzima Novozymes, con el cual se podría obtener $0.02935 \mathrm{Kg}$ de sacarosa, $0.18867 \mathrm{Kg}$ de glucosa por $\mathrm{Kg}$ de $0.0762824 \mathrm{Kg}$ de fructosa por cada $\mathrm{Kg}$ de muestra de arveja seca. De acuerdo con las consideraciones ya mencionadas, se elige el tratamiento 4, que a diferencia de los tratamientos 8 y 5 contiene mayor cantidad de glucosa y fructosa (azúcares reductores), cumpliendo así con el objetivo de esta investigación. 
Basado en los resultados obtenidos, se plantea el uso del hidrolizado de la vaina de arveja fresca (Pisum sativum L.) variedad Santa Isabel, para la fermentación y posterior obtención de alcohol carburante. También es muy factible purificar y concentrar el jarabe obtenido con técnicas de clarificación, sedimentación, floculación, filtrado como las propuestas por Castellanos et.al. (2004) hasta conseguir una especie de la melaza libre de trazas de azufre (si es un hidrolizado proveniente de un tratamiento con ácido sulfúrico), que puede ser usada como materia prima para la elaboración de alimentos para animales; de igual manera, el residuo sólido, debidamente caracterizado, podría ser usado para el mismo fin, ya que después del tratamiento de hidrólisis este material probablemente contenga un valor nutricional que pueda ser provechoso.

Por las características sensoriales observadas en los hidrolizados, se puede pensar en la posibilidad de obtener una bebida, donde se aprovechen las propiedades funcionales y las aplicaciones en la industria que ofrecen los oligosacáridos, como lo mencionan Hernández P. y Jiménez M. (2010) siendo esta la principal razón para pensar en realizar una caracterización del hidrolizado de la vaina de arveja con fin de estudiar más a fondo lo sacáridos encontrados.

Después de haber conocido el comportamiento de la vaina de arveja variedad Santa Isabel en el proceso de sacarificación enzimática, el paso a seguir es trabajar en la optimización del proceso y su estudio de viabilidad económica, teniendo en cuenta las posibles alternativas de uso del hidrolizado, sus limitantes y la relación costo beneficio.

Al observar los datos obtenidos en las pruebas de contenido de material lignocelulósico, se podría decir que dentro de las razones por las que se presentó variación, se encuentran:

- Homogeneidad y tamaño de partícula. El material usado debió haber quedado los más homogéneo posible, pero para ello se necesitaba de un pulverizador.

- La humedad en las muestras. El residuo sólido seco obtenido de los tratamientos se guardó hasta el momento en que se usaron; en este lapso de tiempo pudieron haber tomado humedad del ambiente; algunas de las muestras se tuvieron que transportar a otro laboratorio para ubicarlas en el desecador.

\section{RECOMENDACIONES}

Para próximas investigaciones, en las cuales se desee optimizar el proceso de sacarificación, es conveniente tener en cuenta que se debe enfocar el trabajo en aspectos como: El tamaño de partícula de la muestra y la homogeneidad de la misma.Evaluar la posible interferencia en el proceso de compuestos presentes en la muestras (lípidos, gomas, mucilagos, clorofila y proteínas).

Tiempo, presión y temperatura en la autoclave, así mismo el uso de un equipo que permita controlar estas variables de manera adecuada. 
Ensayos que permita determinar el tiempo óptimo de exposición a la enzima.

Evaluar la estabilidad del hidrolizado en el tiempo (temperatura, $\mathrm{pH}$, luz, degradación, fermentación y solubilidad).

Una opción en la que se puede pensar para reemplazar el ácido en los tratamientos de hidrólisis es trabar con agua en condiciones supercríticas o cercanas al punto crítico (175-250 ${ }^{\circ} \mathrm{C}, 150-250$ atmósferas); ya esta tiene propiedades similares a un ácido (-log $\mathrm{KW}$ $=11$ ), y representa un área de estudio hasta ahora no desarrollada, tal y como lo propone (García, 2006). Claro, esta opción debe ser analizada desde el punto de vista económico, ya que es una técnica mucho más costosa debido a las condiciones en las que se trabaja.

Otra alternativa que se puede estudiar es des lignificar primero la muestra seca de manera similar como se hizo en la determinación de holocelulosa, ya que retirar la lignina facilitaría la acción de la enzima sobre la muestra, teniendo en cuenta lo que concluyen Guarnizo et al. (2009) en su trabajo sobre pre tratamientos de la celulosa y biomasa para sacarificación.

\section{REFERENCIAS BIBLIOGRÁFICAS}

BADUI, D. S. 2006, Química de los alimentos. editorial Pearson Educación, 4ta edición 736 páginas

BERNAL DE RAMÍREZ, I. 1993. Análisis de Alimentos. Academia Colombiana de Ciencias Exactas, Físicas y Naturales. Colección Julio Carrizosa Valenzuela $n^{\circ} 2$. Santafé de Bogotá D.C.

BETTÍN, L. Y QUINTERO D.J. 2010, Estudio de la producción de jarabes glucosados a partir de maltodextrinas empleando dos enzimas comerciales grupo de Bioprocesos. Departamento de Ingeniería Química, Universidad de Antioquia. A.A. 1226. Medellín, Colombia. [En línea] http://redalyc.uaemex.mx/redalyc/pdf/1698/169815396008.pdf (Consultado el 27 de abril del 2010)

BROWING ,1967. Citado por Driss, Alami Saloua Ben. 2010.

CARBALLO,A.; OREA-IGARZAU.; CORDERO-MACHADO E. 2006. Composición Química de Tres Maderas en la Provincia de Pinar del Río, Cuba a Tres Alturas del Fuste Comercial. Parte $\mathrm{N}^{\circ} 1$, Revista Chapingo serie ciencias forestales y del ambiente. vol. 10 número 001. Universidad Autónoma Chapingo. Chapingo México, pp. 57-62. [En línea] http://redalyc. uaemex.mx/redalyc/pdf/629/62910202.pdf. (consultado el 23 de marzo del 2010)

CASTELlANOS D.; CRUZ GUERRERO G.; GONZÁlEZ G. Y RENTERÍA A. 2004. Alternativas para la clarificación del jarabe de glucosa obtenido por hidrólisis enzimática del almidón. Ingeniería e investigación, septiembre, año/vol. 24, número 002. Universidad 
Nacional de Colombia, Bogotá. pág. 8-21. [en línea] http://redalyc.uaemex.mx/redalyc/ pdf/643/64324202.pdf(consultado el 08 de marzo de 2010)

CUERVO, L.; FOLCH J.; QUIROZ R. 2009. Lignocelulosa como fuente de azúcares para la producción de etanol. Centro de Investigación en Biotecnología, UAEM y el Instituto de Biotecnología, UNAM. 2001 Col. Chamilpa, Cuernavaca, Mor. 62209, México. Biotecnología, Vol. 13 No. 311

ECKERT. F, 2008. Ficha técnica Alkozym CPX A, información suministrada por el autor vía correo electrónico, Franz@cht-handes.de, mayo del 2008.

FEDERACIÓN DE CULTIVADORES DE CEREALES Y LEGUMINOSAS (FENALCE). 2010 Revista de la federación de cultivadores de cereales y leguminosas. Fenalce. TPR No. 960-ISSN: 0124-2016. Bogotá D.C. mayo-junio. No. 93. [En línea] http://www. fenalce.org/ (consultado el 25 de noviembre del 2010)

GARCÍA, E.; REYNALDO P. Y GONZÁLEZ J. 2006. Diseño de un proceso para la hidrólisis de residuos lignocelulosicos, Universidad Tecnológica de San Juan del Río, Querétaro, México. [en línea] http://cnea.cicataqro.ipn.mx/cnea/files/BM05P.pdf (consultado el 25 de marzo de 2010)

GONZÁLEZ, B. G. 2010.Conceptualización Química en la cuantificación de la holocelulosa, artículo explicativo.

GUARNIZO, A.; MARTÍNEZ, P.; VALENCIA, H. 2009. Pre tratamientos de la celulosa y biomasa para la sacarificación, scientia et technica, vol. xv, núm. 42, agosto, 2009, pp. 284-289 Universidad Tecnológica de Pereira, Colombia. [en línea] http://redalyc.uaemex. mx/src/inicio/ArtPdfRed.jsp?iCve=84916714053(consultado el 09 de marzo de 2010).

GUMETA, C. 2009. Estudio del secado convectivo y de la extracción de celulosa a través del proceso organosolv a partir de Agave Atrovirens Karw, Instituto Politécnico Nacional. Escuela Nacional De Ciencias Biológicas. México D.F. [En línea] http:/itzamna.bnct.ipn.mx:8080/ dspace/bitstream/123456789/7995/1/115.pdf (consultado el 4 de febrero del 2010).

HERNÁNDEZ P. Y JIMÉNEZ M., 2010. Propiedades funcionales y aplicaciones industriales de los fructo-oligosacáridos Departamento de Ingeniería Química, Alimentos y Ambiental, Revista Temas Selectos de Ingeniería de Alimentos Vol. 4 / No. 1 Universidad de las Américas, Puebla. Sta. Catarina Mártir, Cholula, Puebla. 72810. México [En línea] http:// www.udlap.mx/ofertaacademica/images/filesDIQAA/REVISTA $\% 20$ TSIA $\% 20$ Vol. $\% 20$ 4-1.pdf (Consultado el 20 de marzo del 2010).

HURTADO, H. 2005. (Comunicado personal): Administrador agropecuario de las plantaciones tecnológicas de la universidad católica de Colombia, Kilómetro 21 vía carrera 7. La Caro. Chía, Cundinamarca. Colombia (consulta 30 de noviembre del 2005). Obtenido 
de Hernández C. Ricardo Y López R. Claudia, evaluación del crecimiento y producción de Pleurotus ostreatus sobre diferentes residuos agroindustriales del departamento de Cundinamarca. Tesis Pontificia universidad Javerina.

KEHR, M. Y MERA, M. 2007. Arveja de Vaina Comestible Sugar Snap, Instituto de Investigaciones Agropecuarias Fundación para la Innovación Agraria. Temuco. Chile. [En línea] http://www.inia.cl/medios/biblioteca/boletines/NR34382.pdf (consultado el 24 de febrero del 2010).

LÁZARO, L. YARAUZO, P. 1994. Aprovechamiento de Residuos de la Industria de Conservas Vegetales, Hidrólisis Enzimática. Departamento de Ingeniería Química y T.M.A. Centro Politécnico Superior De Ingeniería, Universidad de Zaragoza. [En línea] http://dialnet. unirioja.es/servlet/articulo?codigo=110293 (consultado el 7 de abril del 2010).

MEJÍA, G.;MARTÍNEZ, C.;BETANCOURT, G.;CASTRILLÓN, C.2007.Aprovehechamiento del Residuo Agroindustrial del Mango Común (Mangifera Indica L.) en la Obtención de Azúcares Fermentables, Ingeniería y ciencias. ISSN 1794-9165, volumen 3, número 6, diciembre del 2007, pp 41-65. [En línea] http://dialnet.unirioja.es/servlet/fichero_ articulo? codigo $=2576982$ \&orden $=0$ (consultado el 23 de marzo del 2010).

MIN, D. Y BOFF J. 2009. El análisis de la grasa bruta, Análisis de los Alimentos, Editorial Acribia, S.A., Zaragoza, España.

NAKAKUKI, T. 2002. Estado actual y futuro del desarrollo de oligosacáridos funcionales en Japón, Articulo Química Pura y Aplicada 74, 1245-1251.

NIELSEN, S. 2007. Análisis de los alimentos, Manual de laboratorio, Editorial Acribia, S.A. Zaragoza, España.

NOVOZYMES, 2008 tomado de http:/www.novozymes.com/en/ServiceMenu/Contact/

SÁNCHEZ, S. 2010. de Aprovechamiento integral del Hesperaloe funifera mediante fraccionamiento de sus componentes, Tesis Doctoral, Facultad de Ciencias. Universidad de Córdoba. [En línea] http://helvia.uco.es/xmlui/bitstream/ handle/10396/3511/9788469329917.pdf?sequence=1 (consultado 10 de febrero del 2010).

SHODEX 1,2011. HPLC Columnes and Instruments 2010-2011. Showa Denk K.K Shodex (Separation and HLPC) Group,5-1, Ogimachi, Kawasaki-ku, Kawasaki, Kanagawa 2100867, Japan.

SHODEX 2, 2010. Analysis of saccharides in food industry, technical notebook No. 2, Showa Denko K.K Shodex(Separation and HLPC) Group,5-1, Ogimachi, Kawasaki-ku, Kawasaki, Kanagawa 210-0867, Japan 
TORTOSA, M. 2007. Extracción de materia orgánica soluble de un compost de Orujo de Oliva de dos fases, Centro de Edafología y Biología Aplicada del Segura, Consejo Superior de Investigaciones Científicas y Universidad de Murcia, España. [En línea] http://digital.csic.es/bitstream/10261/24568/1/Tesina\%20Germ\%C3\%A1n\%20Tortosa. pdf (consultado el 5 de febrero del 2011) TRIANA, C. 2010. Producción de etanol a partir de residuos provenientes del cultico de café, Tesis de Posgrado,Universidad Nacional de Colombia, sede Manizales. [En línea] http://www.bdigital.unal.edu. co/1974/1/cristianfernandotrianacaranton.2010.pdf (consultado 4 de febrero del 2010).

URBANEJA, G.; FERRER JOSÉ, R.; PÁEZ G.; ARENAS DE MORENO, L.; COLINA, G. Y SANDOVAL, L. 1997. Hidrólisis ácida y caracterización de carbohidratos de la pulpa de café, Proyecto No. 0195-94 subvencionado por el Consejo de Desarrollo Científico y Humanístico (CONDES). Venezuela. [En línea] http://www.revfacagronluz.org.ve/ v14_2/v142z010.html (consultado 14 de marzo del 2010).

WISE 1946 Citado por Driss, Alami Saloua Ben. 2010.

YING Y., 2008. Ethanol Production Potential of Acid Pretreated Switchgrass Varieties, A thesis submitted to the Graduate Faculty of North Carolina State University In partial fulfillment of the Requirements for the Degree of Master of Science by Biological and Agricultural Engineering, Raleigh, NC. 